\title{
Tactile, thermal, and electrical thresholds in patients with and without phantom limb pain after traumatic lower limb amputation
}

This article was published in the following Dove Press journal: Journal of Pain Research

20 April 2015

Number of times this article has been viewed

\author{
Shengai $\mathrm{Li}^{1,2}$ \\ Danielle H Melton ${ }^{1,2}$ \\ Sheng $\mathrm{Li}^{1,2}$ \\ 'Department of Physical Medicine \\ and Rehabilitation, University of \\ Texas Health Science Center \\ at Houston, Houston, TX, USA; \\ ${ }^{2}$ Neurorehabilitation Research \\ Laboratory, TIRR Memorial Hermann \\ Research Center, Houston, TX, USA
}

Purpose: To examine whether there is central sensitization in patients with phantom limb pain (PLP) after traumatic limb amputation.

Methods: Seventeen patients after unilateral lower limb amputation secondary to trauma were enrolled. Ten patients had chronic PLP, while the other seven patients had no PLP. Tactilesensation threshold, cold- and warm-sensation thresholds, cold- and heat-pain thresholds, electrical-sensation threshold (EST), and electrical-pain threshold on the distal residual limb and the symmetrical site on the sound limb were measured in all tested patients. Their thresholds were compared within the PLP and non-PLP group, and between the groups.

Results: The novel findings included: 1) electrical-pain threshold was only decreased in the sound limb in the PLP group and there was no difference between two limbs in the non-PLP group, suggesting central sensitization in patients with PLP; and 2) EST was increased on the affected limb as compared to the sound limb within the PLP group, but there were no significant differences in EST between the PLP and non-PLP group. There were in general no significant differences in other tested thresholds within the groups and between groups.

Conclusion: Our results demonstrate central sensitization in the patients with PLP after traumatic limb amputation.

Keywords: central sensitization, pain threshold, human

\section{Introduction}

Phantom limb pain (PLP) refers to a painful sensation of an amputated or deafferented limb. About $60 \%-80 \%$ of patients experience PLP after amputation. ${ }^{1}$ PLP is often described as shooting, stabbing, squeezing, throbbing, and burning, and is primarily localized in distal parts of the missing limb. Severe phantom pain is debilitating to patients, and yet is challenging for physicians who manage it. ${ }^{2}$ PLP has been viewed as a maladaptive response of the nervous system to nerve damage. Mechanisms of phantom pain are not well understood. Possible mechanisms include peripheral and spinal changes and cerebral reorganization after sensory loss from limb amputation; ${ }^{1-4}$ self-consciousness of body image; 5 and persistence of "labelled" somatosensory area after amputation $^{6}$ and central sensitization. ${ }^{7}$

A recent study ${ }^{8}$ showed that local cortical representation was maintained after hand amputation, while chronic phantom pain was related to disrupted interregional connectivity. The altered connectivity was postulated to be driven by nociceptive or top-down inputs. This finding is further supported by another possible mechanism for PLP. It is believed that cognitive-emotional sensitivity and memory play a role in the persistence of phantom pain, which is particularly important if PLP is secondary to a 
traumatic event. ${ }^{9}$ A recent hypermnesia-hyperarousal model is proposed specifically to account for pain persistence and pain sensitization following a traumatizing event. ${ }^{10}$ In case of severe traumatization, such as traumatic limb amputation, intense hypermnesia and hyperarousal are likely imprinted on the victim's memories, and result in central sensitization and pain chronification. This theoretical model is able to account for chronic phantom pain and pain catastrophizing in some patients with a psychological sensitivity, but not in others. ${ }^{11}$ However, comparisons of phantom pain assessment between patients with and without PLP are rarely available in the literature.

Quantitative sensory testing (QST) has been used to compare sensory thresholds between affected and sound limbs of amputee subjects with and without PLP in a number of studies, including mechanical, ${ }^{11-14}$ thermal, ${ }^{11,13,15}$ and electrical $^{16}$ thresholds. Only in one study ${ }^{15}$ were thermal thresholds compared between the affected and sound limbs in subjects with and without PLP separately. No statistically significant difference in all thermal thresholds (cold- and warm-sensation thresholds, cold- and heat-pain thresholds) was found between the affected and sound limbs in each group. Since there is no correlation between responses to noxious heat, cold, and electrical stimulation, a battery of tests is recommended to be used to assess pain sensitivity. ${ }^{17}$

The aim of this study was to examine sensory thresholds (mechanical, thermal, and electrical thresholds) in a cohort of subjects after limb amputation with and without chronic phantom pain. To examine the aforementioned link between traumatization, central sensitization, and PLP, we limited subject enrollment to lower limb amputation after trauma only. Electrical-pain threshold has been used to reflect central sensitization. ${ }^{18-20}$ It was hypothesized that electrical thresholds would be lower in subjects with PLP than in those without PLP after traumatic injury, ie, central sensitization, and that there would be no difference in thermal and mechanical thresholds.

\section{Methods \\ Subjects}

Seventeen amputee subjects (Table 1 for characteristics) volunteered in this experiment. Inclusion criteria included: 1) greater than 18 years of age; 2) unilateral lower limb amputation secondary to trauma, below-the-knee amputation, or above-the-knee amputation (AKA); 3 ) amputation at least 6 months prior to the experiments; and 4) being stable on current pain regime for at least 2 weeks if there is phantom pain. Exclusion criteria included: 1) amputation secondary to other etiologies, such as diabetes mellitus; 2) pain disorders other than PLP (eg, infection); 3) with other severe medical problems, including neurological, pulmonary, cardiovascular, renal, or hepatic disease; 4) with preexisting psychiatric disorders; 5) alcohol or drug abuse; 6) with a pacemaker (contraindication for electrical stimulation); 7) residual limb skin issues, such as inflammation and wound; and 8) diagnosis of neuroma at the residual limb. All subjects gave informed written consent prior to participation. This study was approved by the Committee for the Protection of Human

Table I Characteristics of subjects

\begin{tabular}{|c|c|c|c|c|c|c|}
\hline Subject \# & $\begin{array}{l}\text { Age } \\
\text { (years) }\end{array}$ & Sex & Side & $\begin{array}{l}\text { Level of } \\
\text { amputation }\end{array}$ & $\begin{array}{l}\text { Months after } \\
\text { amputation }\end{array}$ & $\begin{array}{l}\text { Phantom } \\
\text { limb pain }\end{array}$ \\
\hline I & 50 & $\mathrm{~F}$ & $\mathrm{~L}$ & AKA & 42 & $7 / 10$ \\
\hline 2 & 44 & $M$ & $\mathrm{R}$ & AKA & 6 & $7 / 10$ \\
\hline 3 & 58 & $\mathrm{~F}$ & $\mathrm{~L}$ & AKA & 84 & $3 / 10$ \\
\hline 4 & 24 & $\mathrm{~F}$ & $\mathrm{~L}$ & AKA & 49 & $8 / 10$ \\
\hline 5 & 21 & $M$ & $\mathrm{R}$ & $\mathrm{BKA}$ & 33 & No \\
\hline 6 & 49 & $M$ & $\mathrm{~L}$ & BKA & 72 & $6 / 10$ \\
\hline 7 & 51 & $M$ & $\mathrm{R}$ & AKA & 21 & $6 / 10$ \\
\hline 8 & 52 & $\mathrm{~F}$ & $\mathrm{~L}$ & BKA & 144 & $3 / 10$ \\
\hline 9 & 47 & $M$ & $\mathrm{R}$ & AKA & 120 & $2 / 10$ \\
\hline 10 & 49 & $M$ & $\mathrm{R}$ & BKA & 7 & No \\
\hline 11 & 53 & $M$ & $\mathrm{R}$ & AKA & 84 & $5 / 10$ \\
\hline 12 & 40 & $M$ & $\mathrm{~L}$ & BKA & 16 & No \\
\hline 13 & 52 & $M$ & $\mathrm{~L}$ & BKA & 156 & No \\
\hline 14 & 59 & $M$ & $\mathrm{R}$ & AKA & 6 & No \\
\hline 15 & 29 & $M$ & $\mathrm{R}$ & BKA & 8 & No \\
\hline 16 & 44 & $M$ & $\mathrm{R}$ & AKA & 156 & No \\
\hline 17 & 50 & $M$ & $\mathrm{~L}$ & BKA & 8 & $7 / 10$ \\
\hline
\end{tabular}

Notes: Phantom limb pain was reported as the average level of pain over I week prior to the experiments. Visual analog scale was used for phantom limb pain. Abbreviations: AKA, above-the-knee amputation; BKA, below-the-knee amputation; F, female; L, left; $M$, male; R, right. 
Subjects at the University of Texas Health Science Center at Houston and TIRR Memorial Hermann Hospital.

\section{QST}

QST was performed in both affected and contralateral sound limbs, including tactile-sensation threshold (TST), electricalsensation threshold (EST), electrical-pain threshold (EPT), and thermal thresholds. To standardize QST, thresholds on the residual limb were measured at $5 \mathrm{~cm}$ above the distal residual limb, $5 \mathrm{~cm}$ lateral to the midline of the limb, and on the symmetrical site of the contralateral sound limb. After the target areas were localized and marked with a marker, subjects were instructed to stay relaxed in a chair with arms and legs comfortably supported in a symmetrical position. The order of QST was randomized and balanced in two limbs. QST was performed as described in our recent studies. ${ }^{19,20}$

\section{TST}

TST was tested using Von Frey filaments (Touch-Test Sensory Evaluator; North Coast Medical Inc., Gilroy, CA, USA). After a target area was marked and exposed, subjects were instructed to close their eyes. The experimenter pressed different thicknesses of filaments at a $90^{\circ}$ angle against the marked site until they bowed for approximately 1.5 seconds and then removed the filaments. Testing began with the thinnest filament (1.65 grams), then moved to the next thicker monofilament. An explicit response of touch sensation was defined as TST.

\section{EST and EPT}

The same trimmed electrodes were used to measure EST and EPT (electrical stimulator 7SA; Digitimer, Hertfordshire, UK). As previously described, a pair of electrodes (about $2.54 \mathrm{~cm}^{2}$ [1 inch square]) was placed parallel and $5 \mathrm{~mm}$ apart next to each other on a target area. For EST, the intensity of electrical stimulation was started from zero and gradually increased in steps of $0.1 \mathrm{~mA}$ until the subject explicitly felt electrical stimulation. EPT was then measured. The intensity started from the EST and increased in steps of $1 \mathrm{~mA}$ until the subject first felt the electrical stimulation to be painful. To improve consistency among subjects, they were advised that the pain threshold level was equivalent to 1 on the $0-10$ visual analog scale. Three repetitions were made and the average was used for both EST and EPT.

\section{Thermal thresholds}

A Medoc PATHWAY system was used to measure thermal thresholds (warm sensation, cold sensation, heat pain, and cold pain). The established "Limits Full Series" protocol was selected. Briefly, the protocol contains a series of tests in the following order: four tests of cold-sensation threshold (CST), four tests of warm-sensation threshold (WST), three tests of cold-pain threshold (CPT), and three tests of heatpain threshold (HPT). The $30 \times 30$ ATS (advanced thermal stimulation) probe was secured and centered on a target area. All subjects had an education session prior to the protocol. The averaged value was used for each threshold.

\section{Data analysis and statistical analysis}

Descriptive statistics were used. The objective of this study was to compare sensory assessment of both affected and sound legs in amputee subjects with and without phantom pain. Paired $t$-tests were used to compare thresholds between two limbs in all tested patients. The cohort of amputee subjects were further categorized into two subgroups: with and without phantom pain. Paired $t$-tests were again used to compare thresholds within each group. Furthermore, independent $t$-tests were used to compare thresholds between groups if different patterns of results were obtained using data from all subjects or from subgroups. The alpha level required for all statistical significance was set at 0.05 . Data are reported as means \pm standard errors within the text and in the tables and figure.

\section{Results}

Seventeen amputee subjects with AKA or below-the-knee amputation participated in the study. Ten subjects had PLP, while the remaining seven subjects had no phantom pain. Sensory thresholds averaged for all tested subjects, subjects with phantom pain, and subjects without phantom pain are summarized in Table 2. In all tested subjects, EST was significantly greater on the affected limb than on the sound limb $(6.59 \pm 1.03 \mathrm{~mA}$ versus $4.36 \pm 0.47 \mathrm{~mA}, t=0.012)$. Similarly, EPT was significantly greater on the affected limb than on the sound limb $(63.59 \pm 8.07 \mathrm{~mA}$ versus $46.87 \pm 7.01 \mathrm{~mA}$, $t=0.001)$. CPT was significantly decreased on the affected limb as compared to the sound $\operatorname{limb}\left(3.16^{\circ} \mathrm{C} \pm 1.78^{\circ} \mathrm{C}\right.$ versus $\left.7.91^{\circ} \mathrm{C} \pm 2.05^{\circ} \mathrm{C}, t=0.033\right)$. Paired $t$-tests revealed no statistical significance in TST, CST, WST, and HPT in all tested subjects. To examine whether the same pattern of results was obtained in subjects with and without phantom pain, paired $t$-tests were performed for each group (with and without pain) individually. Statistically significant difference in EST and EPT was only found in subjects with phantom pain, but not in subjects without phantom pain. CPT was not significantly different between two limbs in both groups. The same pattern of results was obtained for other thresholds (TST, CST, WST, HPT). 
Table 2 Tactile, electrical, and thermal thresholds were averaged within the PLP group, the non-PLP group, and across all tested patients

\begin{tabular}{|c|c|c|c|c|c|c|}
\hline \multirow[t]{2}{*}{ Thresholds } & \multicolumn{2}{|l|}{ All patients } & \multicolumn{2}{|l|}{ PLP group } & \multicolumn{2}{|l|}{ Non-PLP group } \\
\hline & Affected limb & Sound limb & Affected limb & Sound limb & Affected limb & Sound limb \\
\hline Tactile sensation & 2.98 & 2.91 & 3.24 & 3.21 & 2.61 & 2.47 \\
\hline SE & 0.19 & 0.18 & 0.29 & 0.23 & 0.12 & 0.22 \\
\hline Electrical sensation & 6.59 & 4.36 & 5.51 & 4.07 & 8.13 & 4.79 \\
\hline SE & 1.03 & 0.47 & 0.84 & 0.71 & 2.16 & 0.56 \\
\hline Electrical pain & 63.59 & 46.86 & 53.04 & 31.29 & 78.65 & 69.10 \\
\hline SE & 8.07 & 7.01 & 10.25 & 5.80 & 11.51 & 10.28 \\
\hline Cold sensation & 26.14 & 28.74 & 24.07 & 28.37 & 29.11 & 29.27 \\
\hline SE & 1.86 & 0.60 & 2.97 & 0.88 & 1.00 & 0.76 \\
\hline Warm sensation & 36.21 & 36.00 & 36.69 & 35.69 & 35.52 & 36.43 \\
\hline SE & 0.88 & 0.43 & 1.49 & 0.67 & 0.41 & 0.46 \\
\hline Cold pain & 3.16 & 7.91 & 4.87 & 11.09 & 0.71 & 3.35 \\
\hline SE & 1.78 & 2.05 & 2.94 & 2.90 & 0.46 & 1.83 \\
\hline Heat pain & 46.55 & 46.49 & 45.29 & 45.22 & 48.35 & 48.30 \\
\hline SE & 0.81 & 0.75 & 1.08 & 0.92 & 0.93 & 0.95 \\
\hline
\end{tabular}

Notes: Units for thresholds: $\mathrm{mA}$ for electrical-sensation and -pain thresholds; ${ }^{\circ} \mathrm{C}$ for thermal thresholds; and $\mathrm{g}$ for tactile-sensation threshold.

Abbreviations: PLP, phantom limb pain; SE, standard error.

Independent $t$-tests were then performed for EST, EPT, and CPT for each limb between groups. EST was significantly greater on the affected limb than on the sound limb in the PLP group $(5.51 \pm 0.84 \mathrm{~mA}$ versus $4.07 \pm 0.71 \mathrm{~mA}$, $P=0.010$ ), but both were not significantly different from EST values in the non-PLP group (Table 2 and Figure 1A). The pattern of results was different for EPT. EPT was significantly smaller on the sound limb in the phantom pain group $(31.29 \pm 5.80 \mathrm{~mA})$ than on the other three limbs: 1) the affected limb $(53.04 \pm 10.25 \mathrm{~mA})$ of the PLP group; 2) the affected limb $(78.65 \pm 11.51 \mathrm{~mA})$ of the non-PLP group; and 3) the sound limb $(69.10 \pm 10.28 \mathrm{~mA})$ of the nonPLP group $(P<0.01)$. The EPT values of these three limbs were not significantly different among each other (Figure 1B). These results suggest that the sound limb in the PLP group was sensitized. According to independent $t$-tests, CPT was significantly greater on the sound limb in the PLP group than CPT on the affected and sound limbs in the non-PLP group $(P<0.04)$ (Table 2). As mentioned above, there was no difference in CPT between the affected limb and the sound limb within the PLP group or the non-PLP group.

\section{Discussion}

Tactile, electrical, and thermal thresholds were measured in all 17 subjects after traumatic lower limb amputation with and without phantom pain. The novel findings included: 1) EPT was only decreased in the sound limb in the PLP group, suggesting central sensitization; and 2) EST was increased on the affected limb as compared to the sound limb within the PLP group, but there were no difference in EST between the
A

\section{$\mathrm{EST}(\mathrm{mA})$}

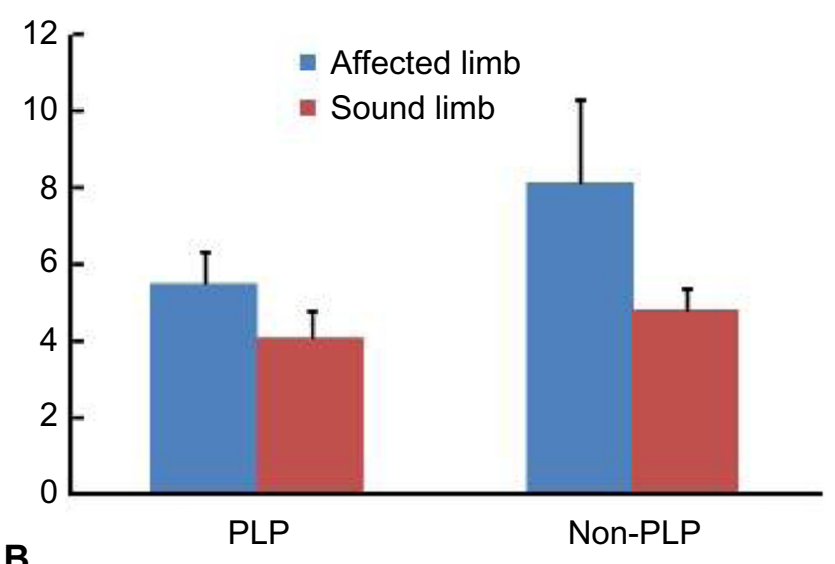

B

EPT (mA)

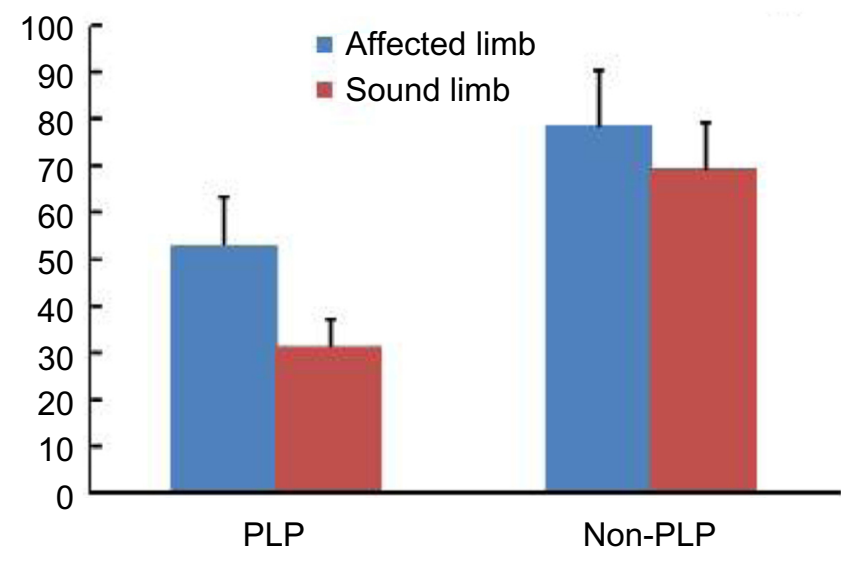

Figure I EST (A) and EPT (B) in the PLP and non-PLP group.

Note: Mean and standard errors are shown.

Abbreviations: EPT, electrical-pain threshold; EST, electrical-sensation threshold; PLP, phantom limb pain. 
PLP group and non-PLP group. Other confirmatory findings ${ }^{15}$ included greater CPT on the sound limb in the PLP group, and no difference in other sensory or pain thresholds (TST, CST, WST, HPT).

To our knowledge, this is the first study that compared mechanical, thermal, and electrical thresholds in both affected and sound limbs in patients with and without PLP. Such comprehensive assessment and comparisons are advantageous. Significant difference in EPT between the affected limb and the sound limb in the PLP group can be accurately interpreted as a significant decrease in EPT on the sound limb in the PLP group after comparing with the EPT values of each limb in the non-PLP group. EPT is reported to have high test-retest reliability. ${ }^{21}$ Furthermore, decrease in EPT has been viewed to reflect central sensitization. ${ }^{18-20}$ The primary significant finding of decreased EPT in the sound limb in the PLP group with no significant difference in other sensory thresholds between two groups suggests central sensitization as a possible mechanism mediating PLP. The finding of selective modulation of EPT was also consistent with our recent studies. ${ }^{19,20}$ We found that EPT was significantly increased after breathing-controlled electrical stimulation (BreEStim), but it was significantly decreased after standard electrical stimulation without changes in TSTs, thermal thresholds, and EST. These studies suggest habituation to experimentally induced pain after BreEStim, but sensitization after standard electrical stimulation.

Our finding of central sensitization with chronic PLP supports the hypothesis of a link between traumatization, central sensitization, and memory-mediated phantom pain, ${ }^{10}$ indicating an important role of central memory processes in chronic phantom pain. ${ }^{9}$ Traumatic injury resulting in limb amputation is usually a single event. The memory of the event could last for the rest of life. When associated with a negative emotional context, PLP could be perceived as aversive, and retriggered by a stressful life event. ${ }^{22}$ On the other hand, our finding is helpful in understanding inconsistent results of phantom pain management by peripheral analgesia. ${ }^{9}$

The finding of a link between central sensitization and chronic PLP suggests that mechanism-based therapeutic interventions could be developed for management of chronic phantom pain. A recently developed noninvasive, nonpharmacological voluntary BreEStim to peripheral nerves has a desensitization effect, as manifested by increased EPT after the interventions. ${ }^{19,20}$ In a recent case report, an AKA patient with chronic shooting phantom pain reported disappearance of PLP after BreEStim treatment. ${ }^{23}$ This could be attributed to the desensitization effect of BreEStim. Future research comparing EPT values in PLP patients before and after BreEStim could further test this mechanism of PLP. In a review article, Moura et al reported that various mind-body interventions for PLP management, including hypnosis, imagery, and visual mirror feedback, are able to offer either temporary or long-term relief, either alone or in combination with conventional therapies. ${ }^{24}$ Our finding supports these mind-body interventions for treatment of PLP. ${ }^{24}$ Moreover, our result suggests that psychotherapy for PLP management focusing on desensitization may bring about better outcomes.

There are a few limitations in this study. Findings of the present study clearly support the role of central sensitization in patients with PLP, but not in patients without PLP. Future studies on its correlation with psychological sensitivity tests are needed to provide further support regarding the connection between PLP and cognitive-emotional sensitivity. Only subjects after traumatic amputations were enrolled to this study. Other etiologies, such as diabetes mellitus and tumor, are major causes of limb amputation. It remains unknown whether the current findings could be generalized to these populations. The findings of no difference in mechanical and thermal thresholds except for CPT are confirmatory and generally consistent with previous studies. ${ }^{11-15}$ The result of greater CPT on the sound limb in the PLP group than in the non-PLP group is likely related to the floor effect of CPT. The Medoc PATHWAY system sets the lower limit for $\mathrm{CPT}$ at $0^{\circ} \mathrm{C}$ for safety reasons. Four out of seven subjects in the non-PLP group and three out of ten subjects in the PLP group reached the lower limit in both affected and sound limbs. Thus, there is a possibility of large variations in the remaining subjects in each group.

\section{Conclusion}

In summary, comprehensive comparisons of sensory thresholds in patients after traumatic lower limb amputation with and without phantom pain demonstrate a primary novel finding of significantly decreased EPT in the sound limb in the PLP group. No difference in other sensory or pain thresholds was found between the PLP group and the non-PLP group. The findings support the idea of central sensitization of PLP. Mechanism-based therapeutic intervention, such as BreEStim, are promising for PLP management, either alone or in combination with conventional therapies. EPT may be used as a measurable variable to quantify phantom pain and to monitor the progress of pain management. 


\section{Acknowledgment}

We thank Liu "Eunice" Yang, MS for her statistical consultation.

\section{Author contributions}

Shengai Li, Danielle H Melton, and Sheng Li conceived and designed the experiments; Shengai Li performed the experiments; Shengai $\mathrm{Li}$ and Sheng $\mathrm{Li}$ analyzed the data; Shengai Li, Danielle H Melton, and Sheng Li discussed and interpreted the data; and Shengai Li, Danielle H Melton, and Sheng $\mathrm{Li}$ wrote and revised the paper.

\section{Disclosure}

The authors report no conflicts of interest in this work.

\section{References}

1. Nikolajsen L, Jensen TS. Phantom limb pain. Br J Anaesth. 2001;87(1): 107-116.

2. Giummarra MJ, Moseley GL. Phantom limb pain and bodily awareness: Current concepts and future directions. Curr Opin Anaesthesiol. 2011;24(5):524-531.

3. Flor H. Phantom-limb pain: characteristics, causes, and treatment. Lancet Neurol. 2002;1(3):182-189.

4. Flor H, Elbert T, Knecht S, et al. Phantom-limb pain as a perceptual correlate of cortical reorganization following arm amputation. Nature. 1995;375(6531):482-484.

5. Ramachandran VS. Consciousness and body image: lessons from phantom limbs, Capgras syndrome and pain asymbolia. Philos Trans $R$ Soc Lond B Biol Sci. 1998;353(1377):1851-1859.

6. Pereira JC Jr, Alves RC. The labelled-lines principle of the somatosensory physiology might explain the phantom limb phenomenon. Med Hypotheses. 2011;77(5):853-856.

7. Woolf CJ. Central sensitization: implications for the diagnosis and treatment of pain. Pain. 2011;152(3 Suppl):S2-S15.

8. Makin TR, Scholz J, Filippini N, Henderson Slater D, Tracey I, JohansenBerg H. Phantom pain is associated with preserved structure and function in the former hand area. Nat Commun. 2013;4:1570.

9. Flor H. Painful memories. Can we train chronic pain patients to 'forget' their pain? EMBO Rep. 2002;3(4):288-291.
10. Egloff N, Hirschi A, von Känel R. Traumatization and chronic pain: a further model of interaction. J Pain Res. 2013;6:765-770.

11. Vase L, Nikolajsen L, Christensen B, et al. Cognitive-emotional sensitization contributes to wind-up-like pain in phantom limb pain patients. Pain. 2011;152(1):157-162.

12. Hunter JP, Katz J, Davis KD. Stability of phantom limb phenomena after upper limb amputation: a longitudinal study. Neuroscience. 2008;156(4):939-949.

13. Hunter JP, Katz J, Davis KD. Dissociation of phantom limb phenomena from stump tactile spatial acuity and sensory thresholds. Brain. 2005; 128(Pt 2):308-320.

14. Braune S, Schady W. Changes in sensation after nerve injury or amputation: the role of central factors. J Neurol Neurosurg Psychiatry. 1993;56(4):393-399.

15. Harden RN, Gagnon CM, Khan A, Wallach G, Zereshki A. Hypoesthesia in the distal residual limb of amputees. PM R. 2010;2(7):607-611.

16. Vase L, Egsgaard LL, Nikolajsen L, Svensson P, Jensen TS, ArendtNielsen L. Pain catastrophizing and cortical responses in amputees with varying levels of phantom limb pain: a high-density EEG brain-mapping study. Exp Brain Res. 2012;218(3):407-417.

17. Janal MN, Glusman M, Kuhl JP, Clark WC. On the absence of correlation between responses to noxious heat, cold, electrical and ischemic stimulation. Pain. 1994;58(3):403-411.

18. Bendtsen L. Central sensitization in tension-type headache - possible pathophysiological mechanisms. Cephalalgia. 2000;20(5):486-508.

19. Li S, Berliner JC, Melton DH, Li S. Modification of electrical pain threshold by voluntary breathing-controlled electrical stimulation (BreEStim) in healthy subjects. PLoS One. 2013;8(7):e70282.

20. Li S, Hu T, Beran MA, Li S. Habituation to Experimentally Induced Electrical Pain during Voluntary-Breathing Controlled Electrical Stimulation (BreEStim). PLoS One. 2014;9(8):e104729.

21. Biurrun Manresa JA, Neziri AY, Curatolo M, Arendt-Nielsen L, Andersen OK. Test-retest reliability of the nociceptive withdrawal reflex and electrical pain thresholds after single and repeated stimulation in patients with chronic low back pain. Eur J Appl Physiol. 2011;111(1): 83-92.

22. Jensen TS, Krebs B, Nielsen J, Rasmussen P. Immediate and long-term phantom limb pain in amputees: incidence, clinical characteristics and relationship to pre-amputation limb pain. Pain. 1985;21(3):267-278.

23. Li S, Melton DH, Berliner JC. Breathing-controlled electrical stimulation could modify the affective component of neuropathic pain after amputation: a case report. J Pain Res. 2012;5:71-75.

24. Moura VL, Faurot KR, Gaylord SA, et al. Mind-body interventions for treatment of phantom limb pain in persons with amputation. Am J Phys Med Rehabil. 2012;91(8):701-714.
Journal of Pain Research

\section{Publish your work in this journal}

The Journal of Pain Research is an international, peer-reviewed, open access, online journal that welcomes laboratory and clinical findings in the fields of pain research and the prevention and management of pain. Original research, reviews, symposium reports, hypothesis formation and commentaries are all considered for publication.

\section{Dovepress}

The manuscript management system is completely online and includes a very quick and fair peer-review system, which is all easy to use. Visit http://www.dovepress.com/testimonials.php to read real quotes from published authors. 